\title{
Microvasculature Of The Urinary Bladder Of The Dog Studied With Light Microscopy, Electron Microscopy And Vascular Corrosion Casts
}

\author{
C.W. Ridner*, R.L. Kao*, and F.E. Hossler** \\ East Tennessee State University, J.H. Quillen College of Medicine, Depts. of Surgery* and \\ Anatomy \& Cell Biology**, Johnson City, TN, 37614 USA.
}

The urinary bladder functions to store and expel urine, and the bladder wall acts as an excellent barrier to the passage of water and electrolytes. These functions require the delivery of nutrients via a rich blood supply to the bladder wall. Yet, ischemia may result from chronic distension or from interruption of the blood flow by inflammation due to injury or neoplasm [1]. Sustained ischemia often compromises the barrier function of the wall [2] and could lead to the loss of the urothelium and even necrosis [3]. Fortunately, complete recovery of the urothelial lining can ensue after relief from the ischemic insult, due partially to the rich blood supply [4]. The gross blood supply to the bladder is well known, but despite its importance, few investigations have been directed towards understanding the three dimensional anatomy of the microcirculation of this organ. In a previous study of the rabbit bladder, we described special features of the microcirculation which might enhance blood flow, despite the normal expansion and contraction of this organ [5]. In the present study we describe the microcirculatory anatomy of the dog bladder using light microscopy, electron microscopy and vascular corrosion casting.

Adult male dogs were anesthetized (pentobarbital and halothane) and anticoagulated (heparin, $500 \mathrm{U} / \mathrm{kg}$ ). The abdominal aorta was exposed and cannulated, the abdominal vena cava was opened, the external iliac arteries as well as the internal pudendal and parietal iliac branches of the internal iliac artery were ligated, and the remaining caudal vasculature was flushed of blood using warm saline. In some cases the bladder volume was adjusted though a range of about 50 to $200 \mathrm{ml}$. The caudal vasculature was then perfused with buffered aldehyde fixative for microscopy or with Mercox resin for vascular corrosion casts. Fixed tissue was embedded in Epon-Araldyte resin for thin and thick sections. Casts were prepared by macerating the tissue in $5 \% \mathrm{KOH}$ and warm water, and cleaned with formic acid and distilled water. Casts were dried by lyophilization, mounted on stubs with colloidal carbon, sputter coated with gold, and viewed by routine scanning electron microscopy. In some cases blood flow was observed by injecting $1 \%$ India ink.

The dorsal wall of the bladder is supplied by the cranial vesicular branches of the umbilical arteries. The basal and lateral walls are supplied by caudal vesicular branches of the urogenital arteries. These vessels spread apically and ventrally and anastomose freely to supply collateral circulation to the apical and ventral bladder walls. The serosal vasculature, especially near the apex, is characterized by large vessels with extensive coiling and abundant venous valves. Coiled arteriole branches ascend to the mucosal surface where they branched abruptly in a Ahydra-like $\cong$ fashion to supply a dense, and freely anastomosing plexus of capillaries just deep to the urothelium. The vasculature of the wall appears to be separated into serosal and mucosal plates joined by the coiled arterioles. The mucosal capillary bed is continuous with similar capillary beds lining the ureters. 
As in the rabbit, the vasculature of the wall of the dog bladder appears well designed to: (1) enhance unidirectional blood flow despite expansion and contraction of the organ (via venous valves, coiled vessels, and collateral circulation); (2) permit blood flow without excessive stress on vessels or vessel kinking during expansion and contraction (via coiled vessels connecting two distinct vascular plates); and (3) provide a rich vascular supply to the urothelium (via an extensive, freely-anastomosing, sub urothelial capillary bed).
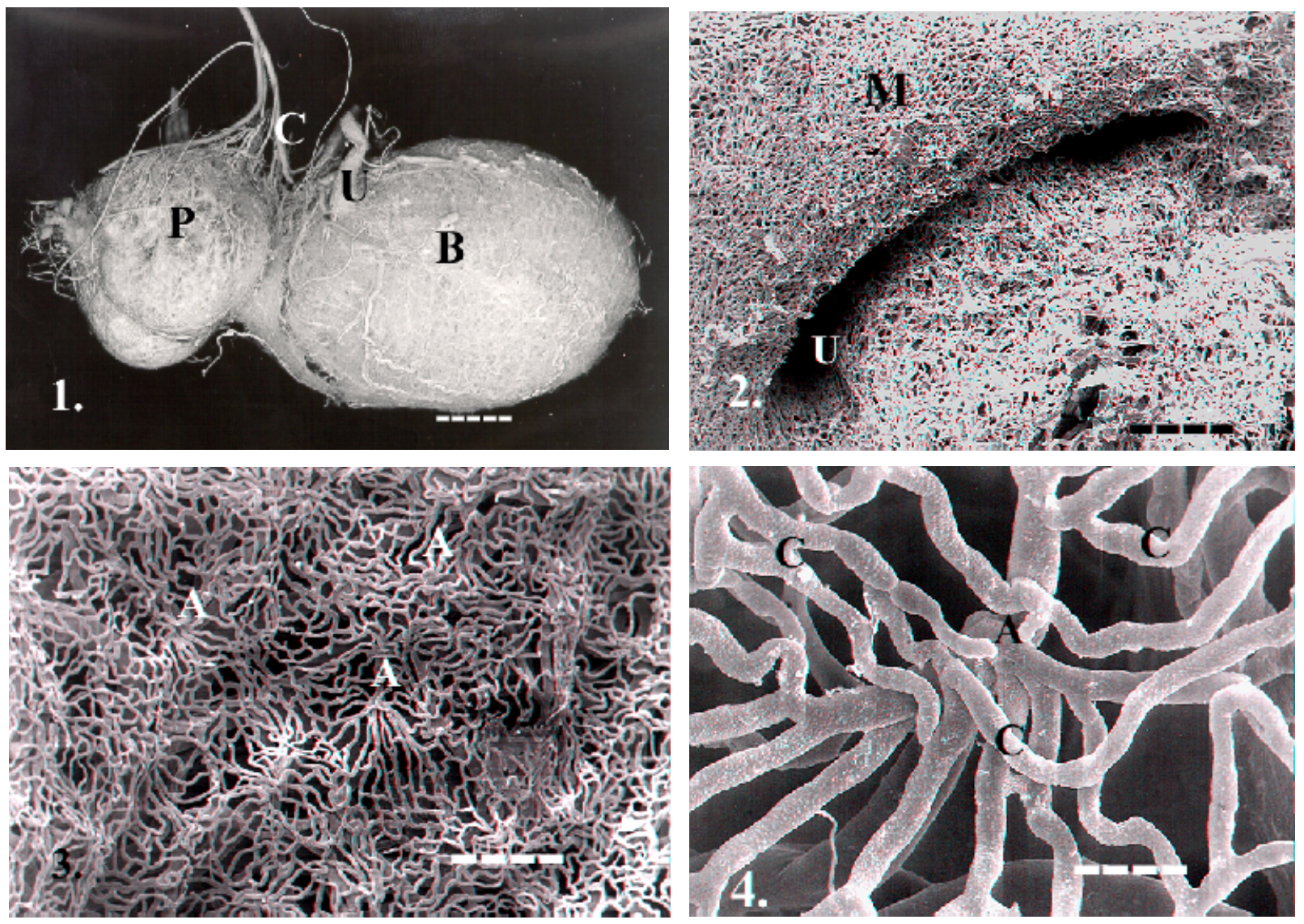

Fig. 1 Vascular corrosion casts of intact dog bladder. B, bladder vasculature; $C$, caudal vesicular artery; P, prostate vasculature; U, vasculature of the ureter. Bar $=10 \mathrm{~mm}$. Fig. 2 Mucosal capillary bed $(\mathrm{M})$ of bladder showing continuity with mucosal capillary bed of ureter; $\mathrm{U}$, opening to ureter. Bar $=500 \mu \mathrm{m}$. Fig. 3 Mucosal capillary bed of bladder. Note multiple capillaries arising from the termini of supplying arterioles (A). Bar $=300 \mu \mathrm{m}$. Fig. 4 Origin of multiple mucosal capillaries $(\mathrm{C})$ from a supplying arteriole $(\mathrm{A})$. Bar $=45 \mu \mathrm{m}$.

\section{References}

[1] M. Dunn, Br. J. Urol. 46 (1974) 67.

[2] R.M. Levin et al., Neurourol. Urodyn., 9 (1990) 269.

[3] F.F. Splann and W.H. Walker, J. Urol. 102 (1969) 429.

[4] H. Gill, et al., J. Urol. 139 (1988) 1350.

[5] F.E. Hossler and F.C. Monson, Anat. Rec. 243 (1995) 438. 